\section{Weniger Exazerbationen mit Indacaterol plus Glycopyrronium}

Der Stellenwert inhalativer Kortikosteroide (ICS) bei chronisch-obstruktiver Lungenerkrankung (COPD) wird intensiv diskutiert. Bei Patienten ohne Exazerbationen begründet die aktuelle Studienlage offenbar keine ICS-Therapie, und bei Exazerbierern ist eine LABA/LAMA-Kombination der LABA / ICS-Gabe vorzuziehen. Zwei Drittel der COPD-Patienten sind mild oder moderat erkrankt und nur $28 \%$ erleiden Exazerbationen, das besagen die Daten der nicht interventionellen DACCORDStudie $^{1}$. Prof. Marek Lommatzsch, Rostock, zitierte sie bei einem Symposium ${ }^{2}$ und ging auch auf die Versorgung der Patienten ein; er betonte: „47\% der Exazerbierer und $42 \%$ der Nicht-Exazerbierer werden mit ICS behandelt." Lommatzsch ging der Frage nach, welcher COPD-Patient wirklich von der ICS-Behandlung profitiert. In der ILLUMINATE-Studie wurden Nicht-Exazerbierer untersucht. Ihre Lungenfunktion wurde durch 1-mal tägliche duale Bronchodilatation mit dem langwirksamem Betamimetikum (LABA) Indacaterol und dem langwirksamem Anticholinergikum (LAMA) Glycopyrronium als Fixkombination ${ }^{3}$ deutlich verbessert. Der Effekt war deutlich stärker als in der Vergleichsgruppe unter dem LABA Salmeterol und dem ICS Fluticason 2-mal täglich ${ }^{4}$. „Die Behandlung nicht exazerbierender COPD-Patienten mit ICS/LABA ist durch die aktuelle Studienlage nicht mehr zu begründen“, folgerte Lommatzsch aus dieser und anderen Studien. „Bei Exazerbierern wirkt die kombinierte Behandlung mit ICS / LABA zwar besser als LABA allein, aber nicht so gut wie LABA/LAMA“, so Lommatzsch weiter. „In der TORCH-Studie 2007 wurde das als sekundärer Endpunkt erfasste Exazerbationsrisiko mit Salmeterol/Fluticason deutlich stärker reduziert als mit einer Salmeterol-Monotherapie. Und in der LANTERN-Studie verringerte Indacate- rol / Glycopyrronium das Risiko wiederum stärker als Salmeterol/Fluticason ${ }^{5}$.“

Dass eine LABA/LAMA-Fixkombination die Lebensqualität steigern kann, zeigte PD Dr. Stephanie Korn, Mainz, anhand der Quantify-Studie: „Mit der Fixkombination Indacaterol/Glycopyrronium, erreichten deutlich mehr Patienten eine klinisch relevante Besserung im Transition Dyspnea Index (TDI) als mit Formoterol/Tiotropium in freier Kombination“, berichtete sie ${ }^{6}$. Das trug dazu bei, dass der Fixkombination ein Zusatznutzen für bestimmte Patientengruppen zugesprochen wurde.

\section{Simone Reisdorf, Erfurt}

${ }^{1}$ Worth H et al. ERS 2014: P929; Vogelmeier C et al. ERS 2014: P930

2 Industriesymposium „Duale Bronchodilatation der symptomatische COPD-Patient im Fokus", anlässlich des 56. Kongresses der Deutschen Gesellschaft für Pneumologie und Beatmungsmedizin, März 2015, Berlin, Veranstalter: Novartis Pharma GmbH

${ }^{3}$ Ultibro $^{\circledR}$ Breezhaler $^{\circledR}$, Novartis Pharma GmbH, Nürnberg

${ }^{4}$ Vogelmeier CF et al. Lancet Respir Med. 2013; 1 : $51-60$

${ }^{5}$ Zhong N et al. ERS 2014: P2815

${ }^{6}$ Buhl R et al. Thorax 2015; 70: 311 -319

In der Open-Label-RAPID-Extensionsstudie $^{2}$ wurden die Teilnehmer im Zeitraum September 2012 bis September 2014 mit dem Alpha-1-Proteinase-Inhibitor weiter behandelt bzw. darauf umgestellt. Eine Zwischenauswertung mit 106 Patienten zeigte, dass der jährliche Abfall der Lungendichte unter Langzeitbedingungen in der Gruppe mit frühem Behandlungsbeginn $(n=53)$ mit den Ergebnissen der RAPID-Studie vergleichbar war und auch die neu eingestellten Patienten (verzögerter Behandlungsbeginn, $n=53$ ) einen vergleichbaren Effekt bei schlechterer Ausgangssituation hatten. Insgesamt sei der frühere Behandlungsbeginn mit einem besseren Effekt assoziiert gewesen, so Herth.

\section{Dagmar Jäger-Becker, Rodgau}

\footnotetext{
${ }^{1}$ Industriesymposium „Seltene Atemwegserkrankungen - häufig übersehen“, anlässlich des 56. Kongresses der Deutschen Gesellschaft für Pneumologie und Beatmungsmedizin, März 2015, Berlin, Veranstalter: CSL Behring $\mathrm{GmbH}$, Marburg ${ }^{2}$ CSL Behring, Data on file
} 